\title{
Star formation activity in the cluster spiral NGC 4254
}

\author{
Krzysztof T. Chyży ${ }^{1}$, Rainer Beck ${ }^{2}$ and Stanisław Ryś ${ }^{1}$ \\ ${ }^{1}$ Astronomical Observatory, Jagiellonian University, Kraków, Poland \\ ${ }^{2}$ Max-Planck-Institut für Radioastronomie, Bonn, Germany
}

\begin{abstract}
With sensitive radio observations of a Virgo Cluster spiral NGC 4254 we are able to decompose thermal and synchrotron radio emission and, based on the thermal component, construct the SFR distribution within the galaxy, unaffected by dust extinction. The mean SFR per unit area is several times higher than in other galaxies of similar Hubble type. Contrary to other Virgo spirals the SFR distribution is not spatially truncated, in concordance with the observed weak HI deficiency. We propose that the SFR enhancement and the observed disturbed morphology of this galaxy can be attributed to tidal triggering by another nearby cluster member.
\end{abstract}

The star formation rate (SFR) is a sensitive indicator of environmental processes that influence cluster-embedded galaxies and their evolutionary state. Multifrequency radio data are especially useful to derive and study the dust-unaffected SFR within galaxies. We investigated the SFR of the Virgo Cluster spiral NGC 4254 for which we performed high-sensitivity radio polarimetric observations at $8.44 \mathrm{GHz}, 4.86 \mathrm{GHz}$, and $1.43 \mathrm{GHz}$ using the VLA and Effelsberg telescopes. With our data we are able to decompose the thermal and nonthermal (synchrotron) radio emission over the whole galaxy by leastsquare fitting of the thermal fraction to the observed radio emission, assuming a constant synchrotron spectral index. The obtained radio thermal distribution is used to predict the extinction-free luminosities in the $\mathrm{H} \alpha$ line, from the beam-independent regions within NGC 4254.

The radio-based predicted $\mathrm{H} \alpha$ luminosities are on average larger than the measured from the observed $\mathrm{H} \alpha$ emission, as expected. The estimated internal extinction seems to be dependent on the region luminosity and its mean value for the whole galaxy is about 0.8 in magnitude. According to our findings the production of stars in the most vivid regions in spiral arms of NGC 4254 is about 20 times higher than in more quiet interarm regions. The mean SFR of the whole NGC 4254 is several times larger than in nearby normal galaxies of similar Hubble type.

The derived enhanced SFR in NGC 4254 is in contradiction to the main trend of reduced SFR in Virgo cluster galaxies compared to isolated spirals. The confrontation of radio thermal emission of NGC 4254 with the optical (R) image does not reveal any reduced thermal emission, even in the southern part, which is most likely to be exposed to the cluster ram-pressure action. This seems to be in concordance with the weak HI deficiency observed in this galaxy. We propose that the enhanced SFR in NGC 4254 is due to tidal triggering by another nearby cluster member. The interaction is able not only to enhance star formation (as observed in some other Virgo Cluster spirals, too) but also to amplify the magnetic field and enhance the radio emission. It also explains the observed disturbed morphology of this galaxy in the optical and radio spectral ranges.

Acknowledgments. This work was supported by the Polish Ministry of Science and Higher Education, grant 2693/H03/2006/31. Based on data observed with the 100-m telescope of the MPIfR at Effelsberg and with the VLA of the NRAO. 